\title{
Approximate MLFMA as an Efficient Preconditioner ${ }^{\dagger}$
}

\author{
Tahir Malas ${ }^{1}$, Özgür Ergül ${ }^{1}$, and Levent Gürel*1,2 \\ ${ }^{1}$ Department of Electrical and Electronics Engineering \\ ${ }^{2}$ Computational Electromagnetics Research Center (BiLCEM) \\ Bilkent University, TR-06800, Bilkent, Ankara, Turkey \\ E-mail: \{tmalas,ergul,lgurel\}@ee.bilkent.edu.tr
}

\section{Introduction}

The solution of the integral-equation problems via the multilevel fast multipole algorithm (MLFMA) has proved to be a successful approach in computational electromagnetics. Among many applications that integral equation methods are applicable, some problems involve open geometries, for which the use of the electric-field integral equation (EFIE) is compulsory. On the other hand, some other applications, e.g., scattering from volumetric targets, involve closed surfaces. The combined-field integral equation (CFIE) is the preferred choice for these problems because it is free from the internal-resonance problem and it provides linear systems that are easier to solve iteratively compared to those obtained with EFIE [1]. To increase robustness and to speed up convergence, preconditioners that approximate the near-field matrix (i.e., incomplete LU or ILU) or its inverse (i.e., sparse approximate inverse or SAI) have been used and shown to be beneficial in moderate size problems [2], [3]. However, as the problem size gets larger, the near-field matrix becomes increasingly sparser and it becomes harder to solve linear systems with these preconditioners in acceptable times.

In this work, we propose a preconditioner that approximates the dense system operator. For this purpose, we develop an approximate MLFMA (AMLFMA), which performs a much faster matrix-vector multiplication with some relative error compared to the original MLFMA. We use AMLFMA to solve a closely related system, which makes up the preconditioner. Then, this solution is embedded in the main solution that uses MLFMA. By taking into account the far-field elements wisely, this preconditioner proves to be much more effective compared to the near-field preconditioners.

In [3], a similar preconditioning scheme has been shown to increase robustness for the solution of large real-life problems employing EFIE. However, we argue that the AMLFMA preconditioner is more efficient both in terms of memory and computational time. In the following sections, we present some of the details of the AMLFMA preconditioner and we provide comparisons with other strong preconditioners to show that not only our preconditioner increases the robustness, but it also decreases the solution time drastically.

\footnotetext{
${ }^{\dagger}$ This work was supported by the Scientific and Technical Research Council of Turkey (TUBITAK) under Research Grant 105E172, by the Turkish Academy of Sciences in the framework of the Young Scientist Award Program (LG/TUBA-GEBIP/2002-1-12), and by contracts from ASELSAN and SSM. Computer time was provided in part by a generous allocation from Intel Corporation.
} 


\section{AMLFMA Preconditioner}

MLFMA performs a fast dense matrix-vector multiplication with a desired accuracy. The maximum error is controlled by the truncation number

$$
L \approx 1.73 k a+2.16\left(d_{0}\right)^{2 / 3}(k a)^{1 / 3}
$$

of the translation function, where $a$ is the cluster size of the level and $d_{0}$ is the accurate number of digits [4]. Both the computational time and the memory requirement of the operations for a level are proportional to $L^{2}$. A less-accurate but cheaper version of MLFMA can be constructed by reducing the number of accurate digits $d_{0}$ as in [3]. However, the truncation number loosely depends on the value of $d_{0}$ for large boxes in the higher levels of MLFMA. For example, for an eight-level problem, if the number of accurate digits is reduced from four to one as in [3], the truncation number of the highest level decreases from 380 to 361, and this corresponds to only $5 \%$ reduction. Hence, as the problem size increases, this approach becomes less effective. Moreover, new sets of arrays are needed for the radiation (receiving) patterns of the basis (testing) functions for the less-accurate MLFMA, and this adds a significant cost to the memory requirement.

In this work, we propose a less-error-controlled but much cheaper version of MLFMA. We call this version AMLFMA, which serves as a preconditioner. For this purpose, we redefine the truncation number for level $l$ as

$$
L_{l}^{\prime}=L_{1}+a_{f}\left(L_{l}-L_{1}\right),
$$

where $L_{1}$ is the truncation number defined for the first level, $L_{l}$ is the original truncation number for the level $l$ calculated by using (1), and $a_{f}$ represents the approximation factor, which is defined in the range from 0.0 to 1.0. As $a_{f}$ decreases from 1.0 to 0.0, AMLFMA becomes less accurate but increasingly cheaper. Since the truncation number of the lowest level is not modified, AMLFMA does not require extra computation load for the radiation and receiving patterns of the basis and testing functions when it is used in conjunction with MLFMA in a nested manner.

To demonstrate the accuracy of AMLFMA, we analyze the relative error in the output vector $\boldsymbol{y}$ for the matrix-vector product $\boldsymbol{y}=\overline{\boldsymbol{A}} \cdot \boldsymbol{x}$, where $\boldsymbol{x}$ is a vector of ones. In Figure 1, we show the number of elements of the output vector $\boldsymbol{y}$ satisfying different error levels. It is interesting to observe that we achieve moderately accurate matrix-vector multiplications, even with $a_{f}=0.2$. This is because, for determining the truncation number, we consider the worst-case scenario for the positions of the basis and testing functions to guarantee the desired level of accuracy. However, there are usually many interactions that can be computed accurately by using lower values for the truncation numbers. Hence, these interactions become useful in the construction of powerful preconditioners, where the accuracy is not critical.

AMLFMA preconditioner is used in an inner-outer solution scheme, where the outer solver should be a flexible Krylov method for the solution of the original linear system. The inner solver is another Krylov method employing AMLFMA, which performs the preconditioning. To obtain maximum efficiency, we need the best approximation with the least possible effort for the inner solution. For AMLFMA 

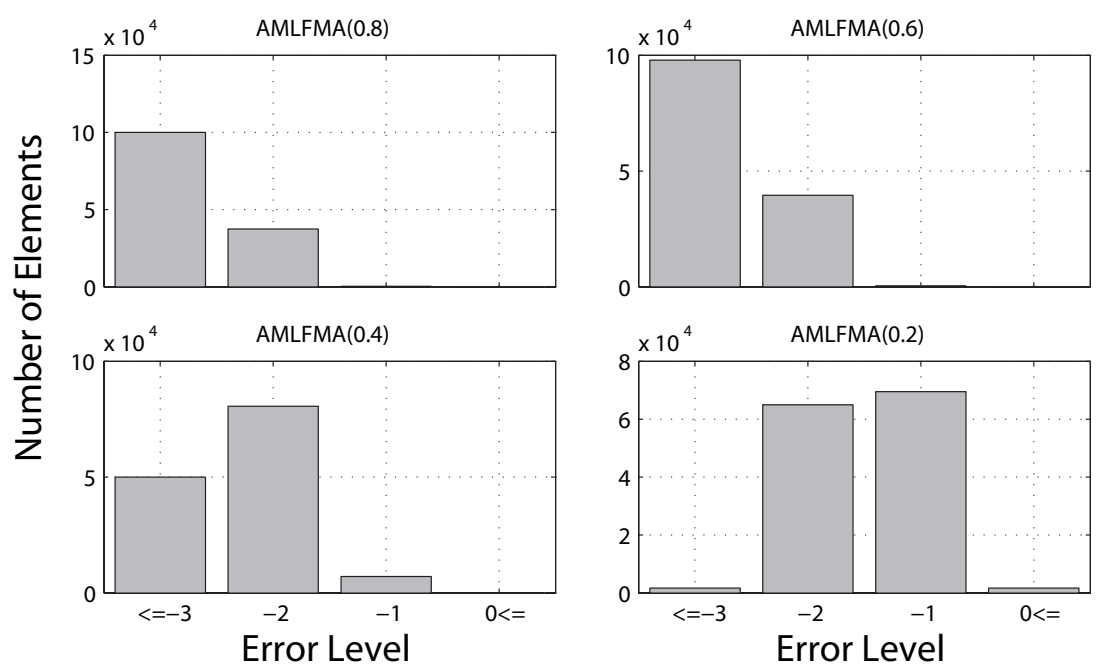

Figure 1: Error levels of AMLFMA with various values of $a_{f}$ for a patch problem of 137,792 unknowns. The reference is MLFMA with three accurate digits.

with $a_{f}=0.2$, almost all elements of the output vector $\boldsymbol{y}$ is computed with less than 0.1 error, while the computation time is significantly reduced. Hence, if we fix the error threshold at 0.1, AMLFMA(0.2) seems to be the best choice. Lower residual errors necessitate a more accurate matrix-vector multiplication, whose computation time cannot be reduced so effectively.

\section{Numerical Results}

We compare AMLFMA preconditioner with SAI and another inner-outer solution scheme called NF/SAI, which proved to be highly successful for EFIE problems [5]. AMLFMA preconditioner uses $a_{f}=0.2$ and the inner solver tolerance is set to 0.1 or a maximum of 10 iterations. The inner solution is accelerated with SAI, for which the near-field pattern is used for the approximate inverse. For NF/SAI, the iterative solution of the near-field matrix provides the preconditioning. This inner solution is also accelerated by SAI and the inner stopping criteria is set to 0.1 residual error or a maximum of only three iterations. For CFIE, we use the familiar block-diagonal preconditioner (BDP) instead of NF/SAI. We use the flexible solver FGMRES in the outer iterations and GMRES in the inner iterations or with SAI and BDP [6]. Numerical solutions are carried out on 32 cores of an Intel quad-core Xeon cluster connected via an Infiniband network.

Table 1 provides the details of the problems. Only the helicopter problem involves a closed geometry for which CFIE is employed. Other problems are modelled with EFIE. Figure 2 presents the plots of the residual norms against solution times. These results demonstrate the outstanding performance of the AMLFMA preconditioner; open-geometry problems are solved two times faster compared to SAI, and 1.5 times faster compared to NF/SAI. The speedup in the helicopter problem is even more impressive; this large problem is solved four times faster compared to BDP and 2.5 times faster compared to SAI. 
Table 1: Information about the problems.

\begin{tabular}{|c|r|r|r|r|}
\hline Problem & $\begin{array}{c}\text { Frequency } \\
(\mathrm{GHz})\end{array}$ & $\begin{array}{c}\text { Size } \\
(\lambda)\end{array}$ & $\begin{array}{c}\text { MLFMA } \\
\text { Levels }\end{array}$ & $\begin{array}{c}\text { Unknowns } \\
N\end{array}$ \\
\hline Reflector Antenna & 1 & 25 & 6 & 356,439 \\
\hline Patch & 96 & 96 & 8 & $3,164,544$ \\
\hline Open Prism & 80 & 139 & 9 & $2,929,136$ \\
\hline Helicopter & 2.66 & 110 & 9 & $2,957,616$ \\
\hline
\end{tabular}
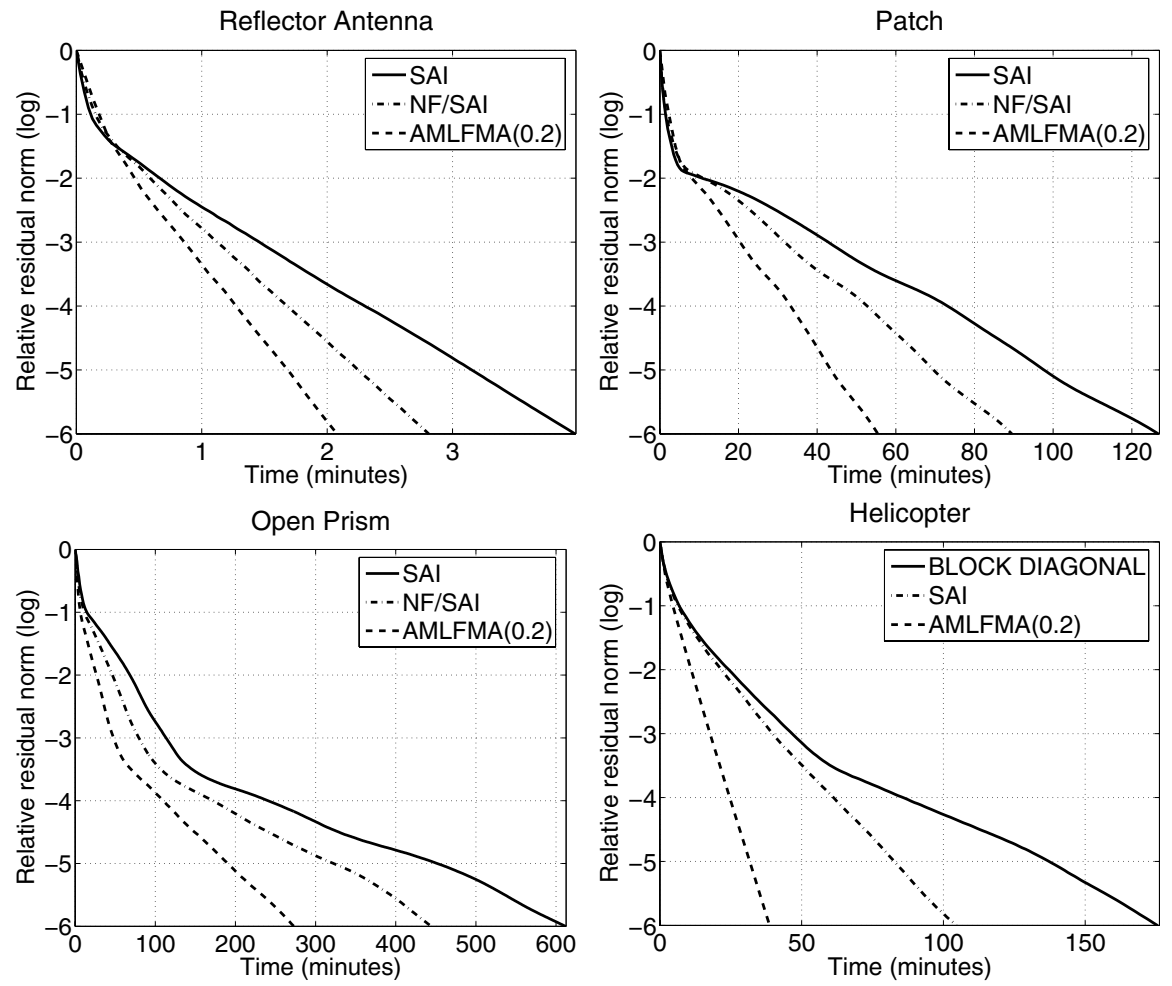

Figure 2: Residual versus time plots for the geometries listed in Table 1.

\section{References}

[1] W. C. Chew, J.-M. Jin, E. Michielssen, and J. Song, Fast and Efficient Algorithms in Computational Electromagnetics. Boston, MA: Artech House, 2001.

[2] T. Malas and L. Gürel, "Incomplete LU preconditioning strategies for MLFMA," in Proc. IEEE Antennas and Propagation Soc. Int. Symp., 2006, pp. 3921-3924.

[3] B. Carpentieri, I.S. Duff, L. Giraud, and G. Sylvand, "Combining fast multipole techniques and an approximate inverse preconditioner for large electromagnetism calculations," SIAM J. Sci. Comput., vol. 27, no. 3, pp. 774-792, 2005.

[4] M. L. Hastriter, S. Ohnuki, and W. C. Chew, "Error control of the translation operator in 3D MLFMA," Microwave Opt. Technol. Lett., vol. 37, no. 3, pp. 184-188, 2003.

[5] T. Malas and L. Gürel, "Strengthening near-field preconditioners using flexible solvers," BiLCEM Tech. Report, Bilkent University, 2006.

[6] S. Balay, K. Buschelman, V. Eijkhout, W. D. Gropp, D. Kaushik, M. G. Knepley, L. C. McInnes, B. F. Smith, and H. Zhang, PETSc Users Manual, Argonne National Laboratory, 2004. 